\title{
Nouvelles stations françaises de Phlébotomes
}

\author{
Par René HOUIN et Jean-Claude BEAUCOURNU
}

L'étude d'une petite collection de Phlébotomes, provenant tous de France métropolitaine, nous a permis de relever quelques éléments nouveaux, qui font l'objet de cette note. Les Insectes examinés ont été capturés par l'un de nous en diverses régions, à l'occasion de séjours estivaux. Il ne s'agit pas en l'occurrence des résultats d'une recherche systématique de Phlébotomes, mais de récoltes effectuées au hasard.

Deux espèces ont été mises en évidence, Phlebotomus perniciosus Newstead 1911 et Phlebotomus ariasi Tonnoir 1921.

\section{PHLEBOTOMUS PERNICIOSUS Newstead, 1911.}

Onze des seize individus capturés appartiennent à cette espèce. Les stations sont les suivantes :

- Bouère (Mayenne) : 1 đo le $1^{\text {er }}$ août 1960,1 \& le 5 août 1960.

Altitude de la station : $68 \mathrm{~m}$. Biotope : taillis.

- Soulgé-le-Bruant (Mayenne) : 1 q le 16 juillet 1960.

Altitude de la station : $70 \mathrm{~m}$ environ. Biotope : Bocage et cultures.

- Montigné-les-Rairies (Maine-et-Loire) : 1 đo le 25 juillet 1965, 1 q le $1^{\text {er }}$ août 1965. Altitude de la station: $60 \mathrm{~m}$. Biotope : bocage humide.

- Juigné-sur-Sarthe (Sarthe) : 2 ơ le 16 juillet 1960, 1 ơ le 5 août 1960, 1 on août 1960.

Altitude de la station : $70 \mathrm{~m}$. Biotope : tocage humide.

— Xaintrailles (Lot-et-Garonne) : 1 o le 5 juillet 1960, 1 ठ le 10 juillet 1960.

Altitude de la station. $150 \mathrm{~m}$ environ. Biotope : vignes et forêts de pins.

Tous les exemplaires examinés ont été capturés à l'intérieur des habitations, soit la nuit, à la lumière électrique, soit le jour, en explorant les coins sombres de la maison servant de gîtes de repos pour les imagos. La présence de cette espèce dans l'Ouest de la France n'est pas une nouveauté, puisqu'elle avait déjà été signalée dans la Mayenne, à Mayenne, et dans le Maine-et-Loire, à Saint-Rémy-la-Varenne, par Langeron et Nitzulescu en 1932. Cependant, à notre connaissance, c'est la première fois que Phlebotomus perniciosus est signalé dans le département de la Sarthe. De 
même, la station de Xaintrailles est la première pour cette espèce dans le Lot-etGaronne.

\section{PHLEBOTOMUS ARIASI Tonnoir, 1921.}

L'espèce est représentée par cinq exemplaires provenant de deux stations :

- Xaintrailles (Lot-et-Garonne) : 1 le 5 juillet 1960, 1 le 28 août 1960.

Altitude de la station: $150 \mathrm{~m}$ environ. Biotope : vignes et forêts de pins.

- Juigné-sur-Sarthe (Sarthe) : 2 le 19 juillet 1960, 1 ơ en septembre 1960.

Altitude de la station: $70 \mathrm{~m}$. Biotope : bocage humide.

L'intérêt de ces deux stations est inégal: la station du Lot-et-Garonne est la première signalée dans le département pour cette espèce, mais il s'agit d'un Phlébotome connu des départements voisins. On peut également noter (ceci étant d'ailleurs vrai pour les deux stations) que ces captures ont été effectuées à l'intérieur des habitations, alors que $P$. ariasi est un Phlébotome essentiellement exophile. Cependant, des exemplaires de cette espèce ont déjà été récoltés dans des constructions habitées, tant par $\mathrm{R}$. M. Nicoli que par nous-mêmes, bien qu'en nombre plus réduit que dans les gîtes extérieurs.

La station de la Sarthe, à l'opposé, présente un intérêt tout particulier. En effet, Phlebotomus ariasi est considéré comme une espèce méditerranéo-atlantique, et les stations les plus septentrionales connues jusqu'à présent étaient celles de la Vienne : Champigny-le-Sec et Poitiers (Rageau, 1948). Du point de vue de leur climat, ces stations sont situées dans une zone atlantique à tendance méditerranéenne [Zone V (IV) du Klimadiagramm Weltatlas], dans laquelle pousse encore le Chêne vert.

Or, la nouvelle station est non seulement beaucoup plus septentrionale que les précédentes, mais encore située dans une zone climatique franchement atlantique (Zone V du Klimadiagramm Weltatlas), impropre à l'implantation de la chênaie d'yeuses. Cette capture doit donc faire réviser nos notions concernant la répartition de l'espèce : celle-ci est capable de vivre dans des régions nettement plus humides et plus froides que celles qui correspondent à la zone du Chêne vert. Non seulement l'espèce y peut exister, mais encore elle s'y maintient pendant plusieurs mois sous forme imaginale, puisque les captures vont du mois de juillet au mois de septembre. Il ne s'agit donc pas là d'une poussée saisonnière unique, correspondant à une période caniculaire. Nous espérons, dans les années à venir, avoir la possibilité d'évaluer la durée de la période où vivent les Phlébotomes à l'état adulte dans cette région.

Il se trouve d'ailleurs que cette nouvelle extension de la zone de répartition de Phlebotomus ariasi corrobore la découverte de l'espèce dans le « Midi meditérranéen » en des points où, non plus du fait de la latitude, mais du fait de l'altitude, le Chêne vert ne pousse plus et est remplacé soit par le Chêne blanc, soit par le Châtaignier.

Dans un autre ordre d'idées, les exemplaires capturés à Juigné-sur-Sarthe présentent encore une particularité méritant d'être notée : si les $q$ apparaissent comme tout à fait normales, le seul ơ capturé, bien que ressemblant par tous les autres caractères 
aux types de l'espèce, possède des organes intromittants un peu différents de ceux qu'il est habituel de rencontrer (fig. 1). Le fait que nous ne possédions qu'un seul exemplaire $\delta$ ne nous permet pas de savoir s'il s'agit là d'une variation morphologique
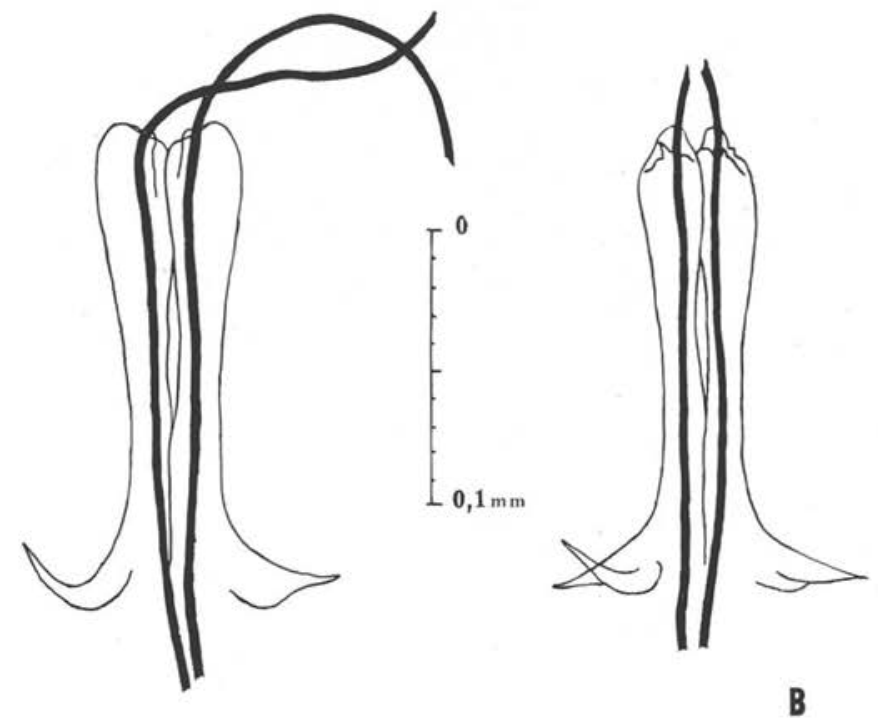

A

Fig. 1. - Organes intromittents de Phlebotomus ariasi Tonnoir 1921: A) P. ariasi typique (provenant de Saillans, Drôme); B) Exemplaire de Juigné-sur-Sarthe

individuelle, ou si nous nous trouvons en présence d'une variété géographique de l'espèce. Nous espérons que la capture de nouveaux exemplaires dans les années à venir nous permettra de résoudre cette question.

\section{Conclusion et Résumé}

L'examen de Phlébotomes adultes provenant de cinq stations différentes de France métropolitaine a permis de reconnaître Phlebotomus perniciosus Newstead 1911 et Phlebotomus ariasi Tonnoir 1921 dans les départements du Lot-et-Garonne et de la Sarthe. Cette dernière localisation est la plus septentrionale qui ait jamais été rapportée pour la seconde espèce. Elle doit faire admettre que Phlebotomus ariasi est capable de vivre dans des régions où ne peut se maintenir le Chêne vert, qui était jusqu'à présent considéré comme l'espèce caractéristique du biotope où se rencontre cet insecte.

Une anomalie morphologique des organes intromittents du seul $P$. ariasi $\delta$ récolté est décrite, sans qu'il soit possible de conclure s'il s'agit d'une variation morphologique individuelle ou d'un caractère permettant de reconnaître une variété géographique. 


\section{Bibliographie}

Langeron (M.) et Nitzulescu (V.), 1932. - Révision des Phlébotomes de France. Ann. Parasit. hum. comp., X (3), 286-294.

Rageau (J.), 1948. - Observations sur les Phlébotomes de la région de Poitiers. Bull. Soc. path. exot., XLI (3-4), 217-222.

WALter (H.) et Lieth (H.), 1960. - Klimadiagramm Weltatlas, 1 vol., Veb. Gustav Fischer Verlag edit., Jena.

(Laboratoires de Parasitologie du C.H.U. de Saint-Antoine, Paris

et de la Faculté de Médecine de Rennes) 